NEWS AND VIEWS

\title{
Double Helix in Single Crystals
}

THE crystal structure of the dinucleotide phosphate adenosyl-3',5'-uridine phosphate (ApU) described by Rosenberg et al. on page 150 of this issue of Nature can be regarded as the "missing link" in nucleic acid stereochemistry. The crystal contains two molecules per asymmetric unit which interact with each other to form a segment of double helix two base pairs long. Each adenine is paired with a uracil and the pairing is of the type proposed for DNA by Watson and Crick ; the helix is righthanded with the two sugar phosphate strands running in opposite directions so that there is an approximate two-fold axis relating the two molecules in the asymmetric unit. This two-fold axis is, however, local to the asymmetric unit and is not a crystallographic symmetry element. The stereochemistry of the sugar-phosphate chain is very similar to that determined for a variety of synthetic and naturally occurring two-stranded RNAs from X-ray diffraction analyses of crystalline fibres.

The similarities between the torsion angles observed in single crystals of nucleic acid components, and those determined in fibre diffraction analyses of nucleic acids themselves have been noted by several workers. This analysis of ApU puts the final touches to such correlations by revealing a structure of a nucleic acid component which not only has the preferred torsion angles characteristic of nucleosides and nucleotides in single crystals but which also looks like a nucleic acid double helix. As the authors rightly claim, it is the double helix at atomic resolution.

Perhaps the most important feature of the structure of ApU is that the base pairing is of the Crick-Watson type. This is the type of base pairing proposed as the basis for molecular recognition in, for example, replication, transcription, codon-anticodon interaction, and for all the structures of naturally occurring and synthetic two-stranded nucleic acids analysed by $\mathrm{X}$-ray fibre diffraction. It is not, however, the form observed in mixed crystals of adenine and thymine (or uracil) bases, nor in the mixed crystal of the nucleosides adenosine and 5-bromouridine. In these crystals pairing through hydrogen bonding of adenine with thymine is observed but it is of the "Hoogsteen" rather than the Watson and Crick type. If the Hoogsteen pairing had been observed in ApU no doubt the analysers of nucleic acid fibres could have retreated and regrouped, but it is a relief to know that this is not necessary.

With the many pearls offered so obviously in the ApU analysis it would be only too easy to overlook many other features of considerable interest. The unit cell contains twenty-six water molecules; when the final analysis locates these accurately their stereochemistry should contribute greatly to understanding of preferred patterns of hydration on nucleic acid double helices and hence to models for hydrophobic stabilization. The binding of ions to nucleic acids is of considerable biological and stereochemical interest. In ApU there is one sodium ion per molecule. The two ions in each asymmetric unit occupy distinct sites. One is complexed between phosphate groups, but the other lies in the minor groove of the DNA helix with octahedral coordination to two of the uracil oxygen atoms and to water molecules. Clearly this latter, rather unexpected type of interaction will stimulate a great deal of physico-chemical interest.

The structure of ApU is given added interest by the simultaneous determination in the same laboratory of the structure of the dinucleotide phosphate guanylyl-3',5'cytidine (GpC) (Day et al., Proc. US Nat. Acad. Sci., 70, 849 ; 1973). GpC also crystallizes as a double-stranded fragment but in this case the two-fold axis relating one $\mathrm{GpC}$ molecule to the other is a crystallographic symmetry element. The base-pairing is of the Watson-Crick type and, as for the structure of ApU, the conformation of the double-helical fragment is very similar to that determined by X-ray analysis of crystalline fibres of two-stranded synthetic and naturally occurring nucleic acids. Again the structure is heavily hydrated with thirty-six water molecules per unit cell.

These studies emphasize the value of single crystal studies of monomers and oligomers in the structural analysis of macromolecules. Increasingly it is being recognized that not only covalent bound geometry but also orientations about single covalent bonds and patterns of intermolecular interaction are strikingly similar in single crystals of macromolecular components to what they are in the macromolecules themselves. As a consequence very precise information obtained from highly refined structures of small molecules can be used in stereochemical analyses of large molecules in which attempts are made to correlate biological, chemical and physical information within a single molecular model.

W. F.

\section{Invoking Black Holes}

ElementaRY accounts of the scientific method often stress the way in which the observations of some unexplained object are built up so that a theoretical model can be inferred from them, as for example in the way the neutrino was invented to explain observations of $\beta$-decay. But there are spectacular counter-examples in which the solution came first and the problem which it fitted was found afterwards. The way in which the positron was found to fit the Dirac theory springs to mind. An astronomical example might be the neutron star. This object was shaken by Fritz Zwicky from what he called his morphological astronomical kaleidoscope, a way of permuting all possible combinations of matter into new objects. It was also invented by Oppenheimer and Volkoff in 1939 as a simultaneous solution of the equation of hydrostatic equilibrium of a self-gravitating mass and an equation of state for a degenerate material made up of neutrons. It was more than 30 years before an astronomical problem was found which appeared to require the neutron star for its solution-the radio and $\mathrm{X}$-ray pulsars.

Of these the $\mathrm{X}$-ray pulsars have provided the most clues 